Bulletin de la Société Royale des Sciences de Liège, Vol. 87, articles, 2018, p. 56 - 61

\title{
Évaluation de la capacité de dissolution des calculs biliaires de Malva sylvestris $\mathbf{L}$.
}

\section{Evaluation of the dissolving capacity of gallstones of Malva sylvestris $\mathbf{L}$.}

\author{
Nadia Boughandjioua Amoura ${ }^{1}$, Nour el Imene Benkirat ${ }^{1}$, Hicham Boughendjioua ${ }^{2 *}$ \\ et Imene Adjailia-Farah ${ }^{1}$. \\ Manuscrit reçu le 30 octobre 2017 et accepté le 22 mai 2018 \\ ${ }^{1}$ Laboratoire de Pharmacognosie, Département de Pharmacie, Faculté de Médecine, \\ Université Badji Mokhtar, Annaba, 23000, Algérie. \\ ${ }^{2 *}$ Département des sciences naturelles, l'école normale supérieure d'enseignement \\ technologique de Skikda (ENSET), 21000, Algérie.
}

\begin{abstract}
Résumé:
Cette étude a pour objectif d'évaluer l'efficacité sur la dissolution des calculs biliaires de cholestérol in vitro d'une plante utilisée en médecine traditionnelle contre la lithiase vésicale; la mauve; Malva sylvestris L. préparée par infusion. Le pouvoir de dissolution de la mauve sylvestre vis-à-vis des calculs biliaires de type cholestérol a été évalué durant une période de huit semaines. La dissolution était significative avec une perte moyenne de masse de $31,7 \mathrm{mg}$ et de $21,49 \mathrm{mg}$ concernant respectivement les calculs de poids faible et moyen à différents grossissements; par ailleurs, la perte était de $0,61 \mathrm{mg}$ pour les calculs de haut poids. Une exposition plus longue pourrait se révéler efficace. De nouvelles expériences sont nécessaires pour confirmer cette hypothèse. Cependant, il paraît difficile aujourd'hui de recommander cette plante pour dissoudre des calculs de cholestérol in situ.
\end{abstract}

Mots clés: cholestérol, plantes médicinales, calculs biliaires, dissolution in vitro, Malva sylvestris.

\begin{abstract}
:
The objective of this study is to evaluate the efficacy on dissolution of gallstones of in vitro cholesterol of a plant used in traditional medicine against bladder stones; the mauve; Malva sylvestris L. prepared by infusion. The dissolving power of the sylvestan mallow towards cholesterol-like gallstones was evaluated over a period of eight weeks. Dissolution was significant with an average mass loss of $31.7 \mathrm{mg}$ and $21.49 \mathrm{mg}$ respectively for the low and medium weight gallstones at different magnification; otherwise the loss was $0.61 \mathrm{mg}$ for the high-weight gallstones. Longer exposure may prove effective. Further experiments are needed to confirm this hypothesis. However, it seems difficult today to recommend this plant to dissolve cholesterol calculations in situ.
\end{abstract}

Keywords: cholesterol, medicinal plants, gallstones, dissolution in vitro, Malva sylvestris.

\footnotetext{
*Adresse électronique pour la correspondance: boughendjioua.hicham@yahoo.com
} 


\section{Introduction}

La prévalence de la lithiase vésicale est en progression de l'ordre de 10-20\% dans les pays occidentaux et de 25-50 \% en Algérie (Maamar, 2003). Pour la traiter ou la prévenir, de nombreuses populations à travers le monde ont recours aux plantes médicinales, ce qui constitue une pratique très ancienne (Klimis, 2009; Macia et al., 2005). La médecine traditionnelle, principalement basée sur les plantes, est mise en œuvre par presque $80 \%$ de la population mondiale (Ramawat et al., 2009) qui utilise des plantes ou leurs extraits contre de très nombreuses pathologies (Fouche et al., 2008; Hi-Guo et al., 2004) y compris contre les calculs. Les données épidémiologiques montrent qu'il peut parfois se former des calculs biliaires, souvent composés de cristaux de cholestérol (dans le $90 \%$ des cas), principalement chez les personnes âgées de 55 à 65 ans. Cependant, cette pathologie n'est symptomatique que chez $20 \%$ des personnes atteinte; elle est associée dans la plupart des cas à une alimentation hypercalorique, trop grasse, entraînant une concentration sanguine trop élevée en cholestérol (Maamar, 2003). De ce fait, l'utilisation des extraits de plantes, dirigés contre cette lithiase cholestéroliques, a fait l'objet de peu d'études en Algérie.

Dans ce travail, nous avons choisi d'étudier l'effet d'extraits de plantes sur les calculs biliaires de cholestérol. Remarquons qu'il est néanmoins possible de traiter les calculs biliaires qui mènent à des symptômes, par les médicaments ou l'utilisation d'ultrasons pour casser les cailloux ou encore la chirurgie afin d'extraire les cailloux ou enfin l'ablation la vésicule biliaire. Cependant, un tel traitement médical est souvent mal toléré avec un taux élevé d'effets secondaires.

Le but de cette étude a été d'évaluer les effets solvants de la mauve sylvestre utilisée en médecine traditionnelle contre la lithiase biliaire. Pour ce faire, nous avons suivi in vivo la variation de masse des calculs au cours de la même période d'incubation que celle utilisée dans une étude marocaine concernant des plantes médicinales proposées contre les calculs de cystine, soit huit semaines.

\section{Matériel et méthodes}

\subsection{Matériel végétal}

L'extrait des feuilles de mauve a été préparé par infusion, pendant 30 minutes, de $3 \mathrm{~g}$ de matière sèche dans $100 \mathrm{ml}$ de solution physiologique bouillante $(9 \mathrm{~g}$ de $\mathrm{NaCl}$ par litre $)$, la solution de $\mathrm{NaCl}$ à $9 \mathrm{~g} / \mathrm{l}$ étant utilisée par ailleurs comme milieu témoin pour apprécier les modifications de masse et/ou de structure des calculs.

Apres filtration, l'extrait a été reparti $(30 \mathrm{ml})$ dans des erlenmeyers en verre (Fig. 1). Les calculs ont été placé dans des compresses stériles (tissu poreux) afin d'éviter tout contact avec le barreau magnétique et mis en suspension dans l'extrait. Ainsi, les calculs biliaires ont été isolés de tout effet mécanique. Chaque extrait a été mis sous une agitation magnétique constante (Hannache et al., 2012).

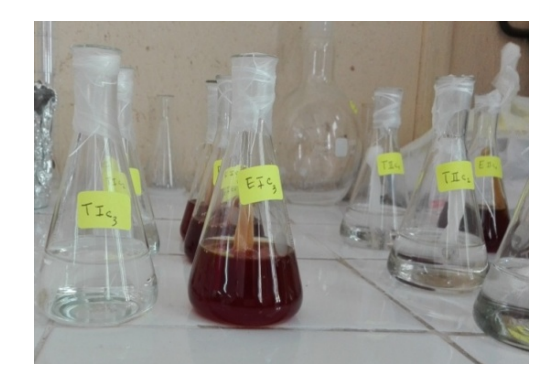

Figure 1: Fiole contenant le calcul inséré dans une compresse stérile immergée. 


\subsection{Calculs biliaires}

Douze calculs biliaires de cholestérol ont été collectés chez deux patients (Guelma, Algérie), sélectionnés par examen en microscopie binoculaire et après analyse par spectrophotométrie infrarouge à transformée de Fourier (FTIR) (Typage morpho-constitutionnel). Leur masse était de $376 \pm 70$, de $114 \pm 10$ et de $80 \pm 50 \mathrm{mg}$ (de haut poids moyen et faible à différent grossissement). Les spectres ont été enregistrés sur un spectromètre FTIR Bruker. La mise en marche ainsi que le traitement des données est assuré par le logiciel OPUS/mentor.

Les calculs ont été laissés au contact des extraits pendant huit semaines. Tous les 15 jours après mesure du $\mathrm{pH}$, ils ont été sortis du milieu expérimental, lavés à l'eau distillée, séchés à $40{ }^{\circ} \mathrm{C}$ durant 16 heures, puis pesés à l'aide d'une balance de précision au $100 \mathrm{e}$ de milligramme (Sartorius) pour évaluer la perte de masse et ensuite replacés dans le milieu pendant les 15 jours suivants. Les pesées ont été réalisées avant la mise en suspension dans les extraits, puis au bout de deux, quatre, six et huit semaines (S0 à S8) (Meiouet et al., 2011).

\subsection{Pourcentage de dissolution}

L'activité des extraits a été évaluée en calculant le taux de dissolution des calculs après séjour dans le milieu expérimental en comparant le poids résiduel des calculs par rapport à leur poids initial avant l'incubation avec l'extrait. Le pourcentage de dissolution a été calculé par la formule :

$$
a \%=\left(W_{\text {initial }}-W_{\text {final }}\right) \times 100 / W_{\text {initial }}
$$

où : $a \%$ est le taux de dissolution du calcul et $W_{\text {initial }}$ et $W_{\text {final }}$ sont les poids du calcul avant et après l'incubation avec les extraits de plantes.

\section{Résultats}

\subsection{Cinétiques de dissolution}

La capacité de dissolution des calculs de différent poids et grossissement par l'extrait durant huit semaines est illustrée par les Fig. 2,3 et 4. Les cinétiques de dissolution semblent être différentes selon le poids des calculs. Durant les deux premières semaines, la masse des calculs de faible poids et moyen a légèrement varié entre deux et quatre semaines, le taux de dissolution a commencé à augmenter et diminue progressivement entre quatre et six semaines. Les résultats se sont confirmés après huit semaines, ces extraits de plante montrant un effet sur la dissolution des calculs plus marqué sur les calculs de poids faible et moyen, exprimé par une perte de masse importante par rapport aux calculs de haut poids qui était négligeable; $(0.61 \mathrm{mg})$ et surtout à la solution de $\mathrm{NaCl}$. Toutefois, cette perte de masse (pour les solutions témoins) n'a pas atteint le seuil de significativité sur la période de huit semaines fixée pour l'expérience.

En ce qui concerne la perte moyenne de masse des calculs, on peut constater que la perte de masse était de 21,49 mg à la fin de l'expérience (huit semaines) pour les calculs de poids faible et de $19.4 \mathrm{mg}$ pour les moyens, contre respectivement 5,04 et 5,17 mg pour la solution de $\mathrm{NaCl}$; une perte de masse moyenne pour l'ensemble des expériences a été de 41,50 mg.

En ce qui concerne le $\mathrm{pH}$ du milieu, la valeur initiale était de 5,9 pour la solution saline et de 6,7 pour l'extrait. Nous avons observé une diminution initiale du $\mathrm{pH}$ pendant les quatre premières semaines de l'expérience pour l'extrait de la plante ainsi que pour la solution témoin. 
Bulletin de la Société Royale des Sciences de Liège, Vol. 87, articles, 2018, p. 56 - 61
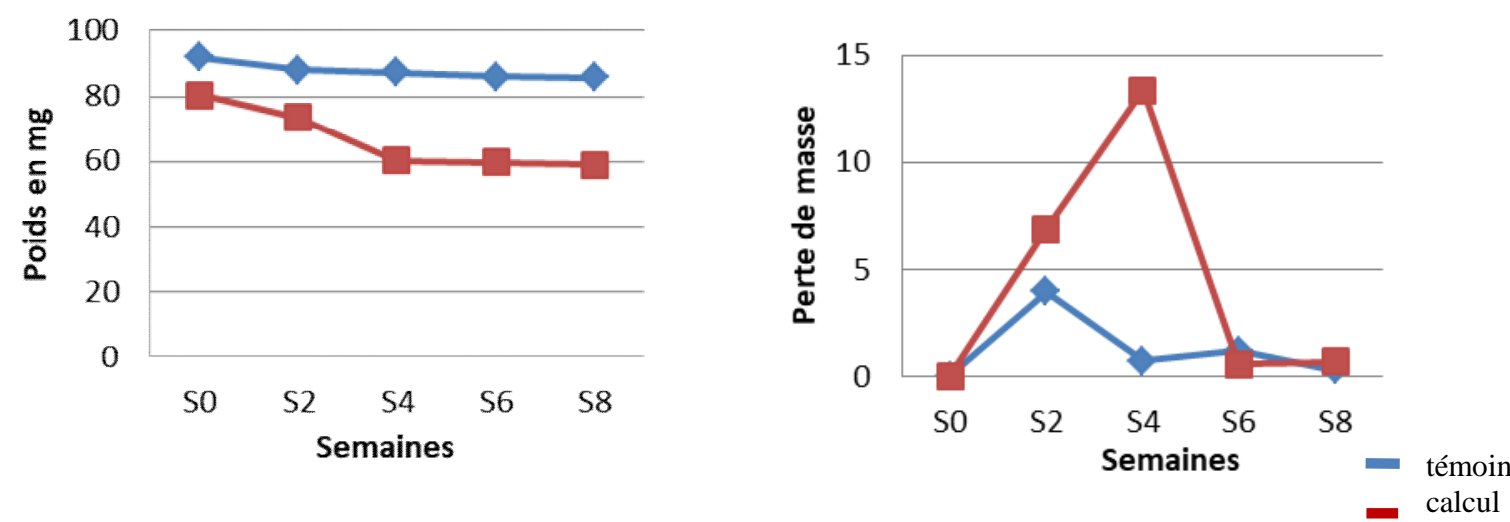

Figure 2: Perte de masse du calcul de faible poids et de son témoin
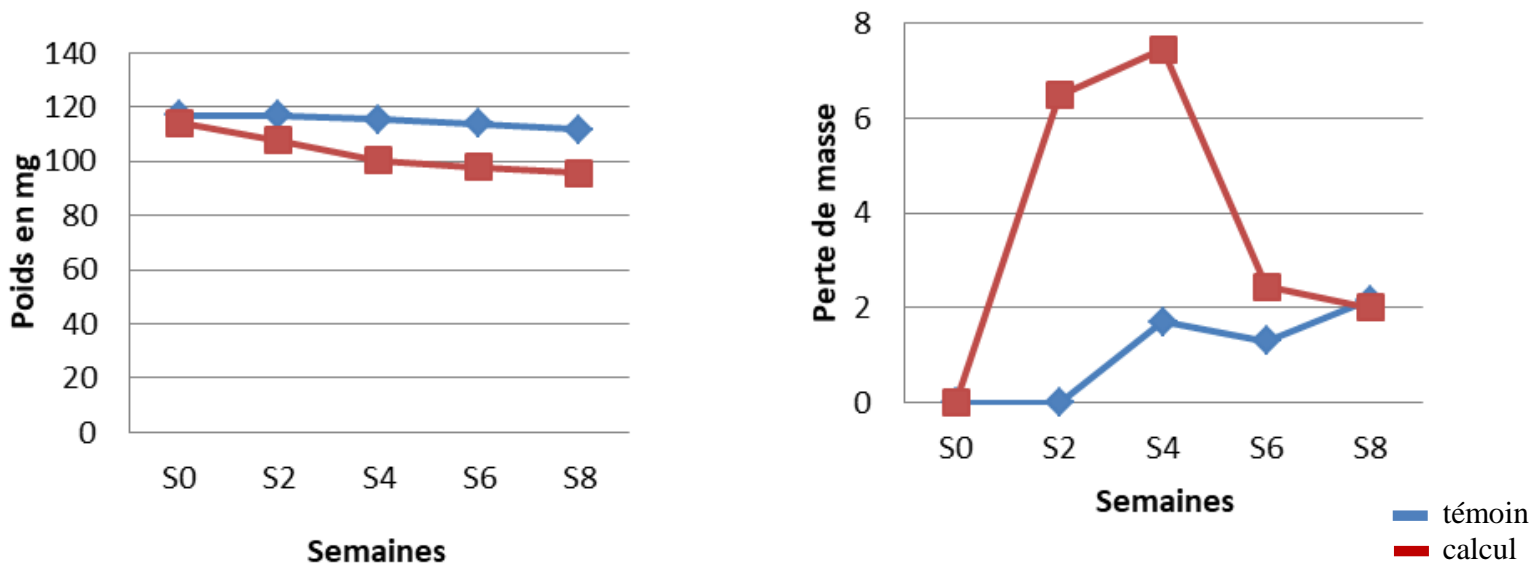

Figure 3: Perte de masse du calcul de poids moyen et de son témoin.
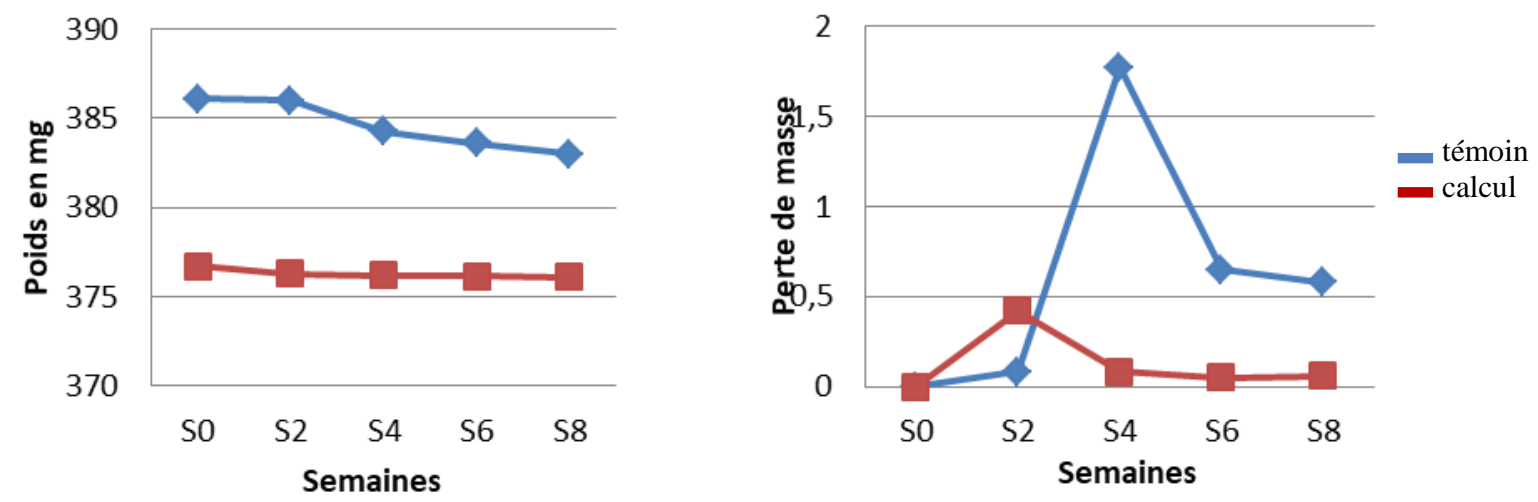

Figure 4: Perte de masse du calcul de haut poids et de son témoin. 


\section{Discussion}

La plante sélectionnée pour notre étude n'a pas été choisie aléatoirement. Elle est utilisée actuellement en Algérie pour le traitement médical des lithiases cholestéroliques ou pour prévenir leur formation. Nous avons donc étudié in vitro l'effet de l'extrait de la mauve sylvestre sur la dissolution de calculs biliaires de cholestérol et nous avons choisi la technique de l'évaluation de la perte de masse pour suivre les changements éventuels dans le poids des cristallites permettant d'affirmer l'existence d'interactions entre les extraits de la plante et les calculs. L'examen des Fig. 2,3 et 4 montre que la pente de la courbe de dissolution obtenue pour les calculs de faible et moyen poids s'accentuait fortement et atteint son max vers la quatrième semaine, puis diminue progressivement au bout de huit semaines. Celle du calcul de haut poids est totalement différente augmente légèrement vers la $2^{\text {éme }}$ semaine puis diminue et devient négligeable à la fin de l'expérience. Nos résultats n'ont pas permis de voir une différence significative entre les extraits et la solution témoin en ce qui concerne la perte de masse des calculs sur la période expérimentale choisie. Cependant, on peut constater que l'importance de la perte de masse est proportionnelle aux poids du calcule (faible, moyen et gros).

En ce qui concerne le $\mathrm{pH}$ du milieu, on constate que les valeurs du $\mathrm{pH}$ obtenue avec la solution saline et l'extrait de plante sont très voisines, suggérant que les effets des extraits de plante sur la perte de masse des calculs ne sont pas liés au pH mais à la composition chimique de l'extrait de plante.

\section{Conclusion}

L'évaluation des résultats par la perte de masse des calculs comparativement à une solution de référence de $\mathrm{NaCl} 9 \mathrm{~g} / \mathrm{l}$ n'a pas permis de mettre en évidence un effet solvant significatif de cet extrait de plante sur la période expérimentale choisie. Au vu des courbes de perte de masse des calculs testés, ce manque de significativité s'explique probablement par une durée de contact insuffisante (durée du traitement traditionnelle est de 12 semaine). De ce fait, malgré l'utilisation de la mauve en médecine traditionnelle pour le traitement de la lithiase biliaire, on ne peut recommander, dans l'état actuel de nos connaissances, l'utilisation de cette plante pour le traitement ou la prévention des calculs biliaires. Des essais complémentaires seront nécessaires pour objectiver un bénéfice scientifiquement démontré. Cependant, cette plante pourrait avoir un intérêt, qui reste à évaluer, pour la prévention des récidives.

\section{Références}

Fouche G., Cragg G.M., Pillay P., Kolesnikova N., Maharaj V.J., and Senabe J. (2008). In vitro anticancer screening of South African plants. Journal of Ethnopharmacology, 119: 45561 .

Hannache B., Bazina D., Boutefnouchet A., and Daudond M. (2012). Effet des extraits de plantes médicinales sur la dissolution des calculs rénaux de cystine in vitro: étude à l'échelle mésoscopique. Progres en Urologie, 22: 577-582.

Hi-Guo Z., Xiao-Ling Y., and Sui-Sheng X. (2004). Application of Chinese traditional medicine in kidney transplantation. Chinese Journal of Integrative Medicine,10: 3104.

Klimis G. (2009). Medicinal herbs and plants in Homer. In: Paipetis SA, editor. Science and technology in Homeric epics. Collection History of mechanism and machine science, 6(VII). Berlin: Springer, 283-91. 
Maamar S. (2003). Journal algérien de chirurgie, 3:16-17.

Macia M.J., Garcia E., and Vidaurre P.J. (2005). An ethnobotanical survey of medicinal plants commercialized in the markets of $\mathrm{La} \mathrm{Paz}$ and El Alto, Bolivia. Journal of Ethnopharmacology, 97:337-50.

Meiouet F., El Kabbaj S., and Daudon M. (2011). Étude in vitro de l'activité litholytique de quatre plantes médicinales vis-à-vis des calculs urinaires de cystine. Progres en Urologie, 21: 40-7.

Ramawat K.G., Dass S., and Mathur M. (2009). The chemical diversity of bioactive molecules and therapeutic potential of medicinal plants. In: Ramawat KG, editor. Herbal drugs: ethnomedicine to modern medicine. Berlin: Springer, 7-32. 